\title{
The secular evolution of M83 central bulge
}

\author{
H. Dottori ${ }^{1}$, R. J. Diaz ${ }^{2}$, M. P. Agüero ${ }^{3}$, D. Mast $^{3}$ and I. Rodrigues ${ }^{4}$ \\ ${ }^{1}$ Instituto de Física, UFRGS,cp: 15051, cep: 91501-970, Porto Alegre, Brazil \\ email: dottori@if.ufrgs.br \\ ${ }^{2}$ Gemini Observatory, Southern Operations Center, Chile \\ CASLEO, CONICET, Argentina \\ e-mail: rdiaz@gemini.edu \\ ${ }^{3}$ Observatorio Astronómico de Córdoba, Laprida 854, Córdoba, Argentina \\ ${ }^{4}$ UNIVAP, São José dos Campos, Brazil
}

\begin{abstract}
The luminosity profile of M 83 bulge can be traced by a de Vaucouleurs' law between $\approx 200 \mathrm{pc}$ and $\approx 800 \mathrm{pc}$. The inner part can be fitted by a $n=-1 / 2$ Sérsic profile. Also the IR $(J-K)$ color shows difference between the periphery and the central part of the bulge, both properties indicating the presence of a pseudobulge. Previous Gemini-S 3-D, $\mathrm{Pa} \beta$ spectroscopy of the central $\approx 5^{\prime \prime} \times 13^{\prime \prime}$ revealed spider like diagrams indicating disk like motion around three extended masses identified respectively with the optical nucleus (ON), with the center of the bulge isophotes, similar to the $\mathrm{CO}$ kinematical center (KC), and with a condensation hidden at optical wavelengths $(\mathrm{HN})$, coincident with the largest lobe in $10 \mu \mathrm{m}$ emission, most probably a cannibalized satellite. Numerical simulations show that they suffer strong evaporation and they would merge engulfing also the star forming arc in few hundred Myr, increasing the mass at the kinematical center by a factor o five or more. Upper mass limit of putative Black Holes associated to $\mathrm{ON}, \mathrm{KC}$ and $\mathrm{HN}$ are a few ten thousand to a million solar masses. GMOS+Gemini imaging and spectroscopy of a chain of radio sources has yield no optical high redshift counterparts. This radio sources are aligned with $\mathrm{ON}$, neither associated to SN nor to HII regions and might point to an older similar phenomenon, which left behind a kick-off spur.
\end{abstract}

Keywords. galaxies: individual (M83), galaxies: kinematics and dynamics, black hole physics

\section{Introduction}

M 83 and Cen A are the largest galaxies in one of the most active regions in the nearby sky, the Hydra-Centaurus group. Processes of mergers as well as galactic inner buildup are evident in both galaxies. M 83 is a unique case for this type of studies, because of the richness of phenomena occurring at the bulge center. Indeed, the condensation traditionally recognized as its nucleus $(\mathrm{ON})$ is observable at optical wavelengths and is off-centered by $4^{\prime \prime}$ to the NE with respect to the center of the external $\mathrm{K}$ band isophotes of the bulge which can be fitted by a de Vaucouleurs' law in an annular region between $\approx 10^{\prime \prime}$ and $40^{\prime \prime}$ (Jensen et al. 1981). Thatte, Tecza \& Genzel (2000) demonstrated that the bulge center host an obscured condensation $(\mathrm{KC})$. Assuming a virialized system, they derived comparable masses $M_{K C} \approx M_{O N} \approx 13.2 \times 10^{6} M_{\odot}$ inside $5.4 \mathrm{pc}$ in a cluster or a super massive core. From CO interferometry Sakamoto et al. (2004) found steep velocity gradients across $\mathrm{ON}$, and determined a mass of $3 \times 10^{8} \mathrm{M}_{\odot}$ within a radius of $40 \mathrm{pc}$, nevertheless, the spatial resolution in CO observations is of the order of $3^{\prime \prime}$ and inhibits conclusions about the precise position of the rotation center (see Diaz et al. 2006). Elmegreen et al. (1997) determined for ON a photometric mass of $M_{O N} \approx 4 \times 10^{6} M_{\odot}$ (Hot Spot 1 in their table 4). More recently, Mast, Diaz \& Aguero (2006) demonstrated the existence of a third condensation $(\mathrm{HN})$, also hidden at optical wavelengths, which 

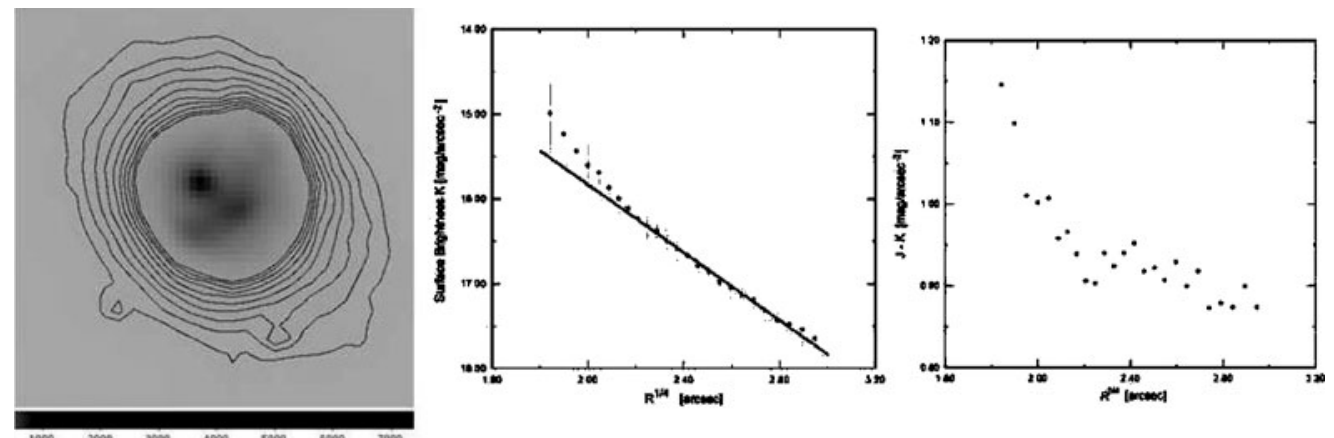

Figure 1. a) 2-mass $\mathrm{K}$ band $67^{\prime \prime} \times 69^{\prime \prime}$ central field showing external bulge isophotes. b) de Vaucouleurs fit well inside $10^{\prime \prime}<R<40^{\prime \prime}$. c) $(J-K)$ color inside $R<40^{\prime \prime}$. Note the change in $K$ and $(J-K)$ inside $R<10^{\prime \prime}$.

coincides with the maximum of continuum emission at $6 \mathrm{~cm}$ and $10 \mu \mathrm{m}$ of a giant cloud (Telesco 1988) $7^{\prime \prime}$ to the WSW of KC. Diaz et al. (2006b) propose that HN is a small galaxy been cannibalized.

We discuss here in more detail the IR structure and colors of M83 central Kpc, as well as the effort made in detecting spurs of previous episodes similar to the undergoing merge inside the central $300 \mathrm{pc}$ of this interesting bulge.

\section{Observations}

$\mathrm{K}$ and $\mathrm{J}$ images of the 2-MASS Large Galaxies Atlas (Jarret et al. 2003) have been used to study $K$ brightness and $(J-K)$ color profiles, with better spatial resolution than previous studies. The kinematics at the bulge center was studied at excellent conditions and with high spatial resolution 2-D spectroscopy with CIRPASS, (Parry et al. 2004) installed on the GEMINI-S telescope in March 2003. The IFU data, (partially presented by Diaz et al. 2006 and Dottori et al. 2007) have got a sampling of $0.36^{\prime \prime}$ (7.8 pc) in an elliptical array with size $4.7^{\prime \prime} \times 13^{\prime \prime}$.

On June 2007 GMOS-S at GEMINI-S has been used to check for the true nature of the radio source Maddox 28: is it at the distance of M 83 or is it a QSR at $z=2$, as suggested by Maddox et al. (2006). We performed broad and narrow band imaging in the range $500 \mathrm{~nm}$ to $950 \mathrm{~nm}$ centered at the position of radio-source 28 and with average seeing of $0.8^{\prime \prime}$. Spectroscopy in the same range spectral was performed with a $1.5^{\prime \prime}$ long slit along the line of radio sources shown in Figure 2 with similar seeing and in poor weather queue, being $3600 \mathrm{~s}$ the approximate effective exposure time. The observation details will be presented elsewhere (Dottori et al., in preparation).

\section{Bulge and pseudobulge brightness and color}

As already determined by Jensen et al. (1981) and Adamson, Adams \& Warwick (1987), higher resolution 2-mass photometry shows that a de Vaucouleurs' law can be traced to the external part of the M 83 bulge in the interval $200<R<800$ pc (Figure 1). The color $(J-K)$ is also uniform in this region, becoming redder inside $R=200 \mathrm{pc}$. The isophotes of the de Vaucouleurs' bulge are circular at $R \approx 200$ pc but they become elliptical outwards, with major axis aligned with the bar. In spite of the non-regular light distribution, the inner region can be described by a Sérsic profile $n=-1 / 2$. This region with characteristic of pseudo-bulge is suffering a process of merger that in few hundred 


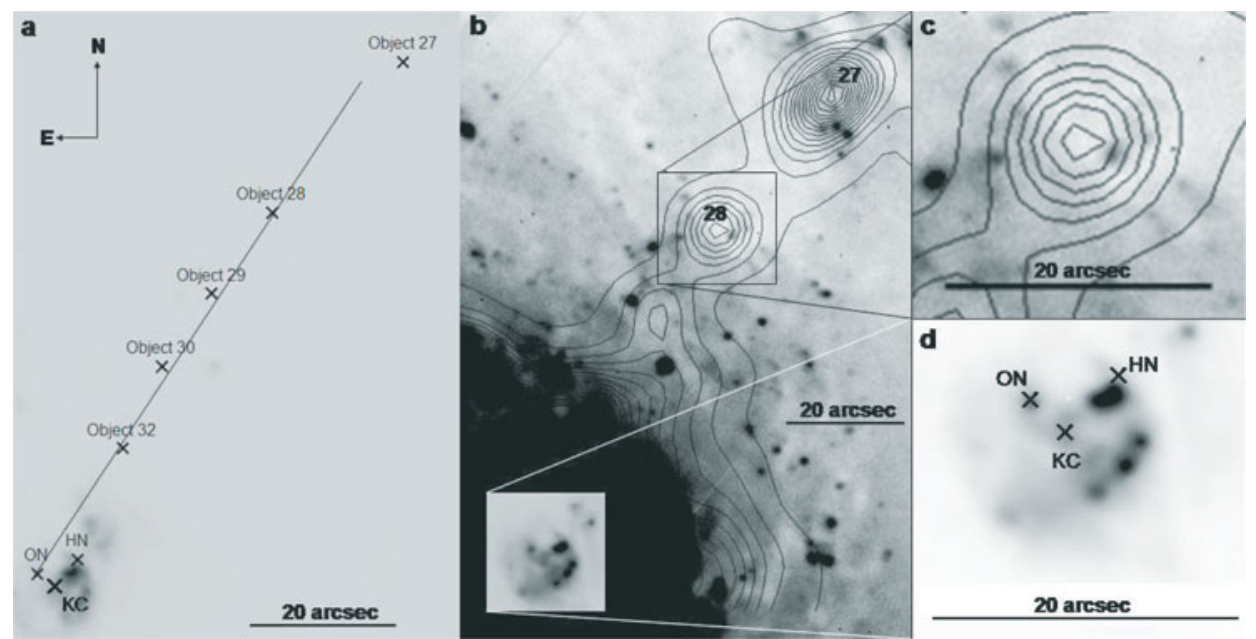

Figure 2. a) Chain of radiosources aligned with ON. b) Radiomap superposed to the $500 \mathrm{~nm}$ to $950 \mathrm{~nm}$ co-added image. c) Object 28 detail: no optical counterpart is found, and the nearest objects depict local HII region line emission. d) Bulge center map showing ON, KC and HN.

Myr will multiply by a factor of five or so the mass of KC, by adding the ones of ON, the intruder HN and that of the arc of HII regions.

\section{Pseudobulge kinematics}

Three spider diagrams can be well differentiated on the complex 2-D $\mathrm{Pa} \beta$ isovelocity contours. They identify the optical nucleus (ON), the bulge center (KC) and the strongly absorbed hidden nucleus (HN). A detailed description of the M 83 central region astrometry can be found in Diaz et al. (2006a). KC shows an inclination of $68^{\circ} \pm 10^{\circ}$ and the $\mathrm{HN}$ inclination is $50^{\circ} \pm 10^{\circ}$. The masses derived by Dottori et al. (2007) are $\approx 4 \times 10^{6}$ $\mathrm{M}_{\odot}, 60-70 \times 10^{6} \mathrm{M}_{\odot}$ and $30-40 \times 10^{6} \mathrm{M}_{\odot}$, respectively.

Upper mass limit for putative $\mathrm{BH}$ associated to each one of the condensations are $(\mathrm{ON})$ $\sim 10^{6} / \sin (i) \mathrm{M}_{\odot}$, and for $(\mathrm{KC})$ and $(\mathrm{HN}) 0.2-1.0 \times 10^{6} / \sin (i) \mathrm{M}_{\odot}$. The kinematical data allowed also to constrain the knots relative motion. Numerical simulations, show that $\mathrm{ON}, \mathrm{KC}$ and $\mathrm{HN}$ will merge, forming a single massive core in 50-100 Myr, an that the HII regions will also be swallowed in a few hundred Myrs.

The short dynamical timescales of this merger make of this phenomenon a unique one (probability less than 1\% per galaxy). Since the Centaurus-Hydra Group to which M83 belongs together with Cen $\mathrm{A}$ is so rich in activity, it is natural to rise the question on why should not have previously occurred a similar phenomenon in M 83 .

\section{A signature of a previous accretion?}

The FII type radio-source 28 in Maddox et al. (2006) list, projected onto M 83 disk, presents boosted radio-lobes (objects 27 and 29) strongly aligned with the galactic bulge and other radio-sources at a projected distance between 1 and $2 \mathrm{kpc}$ (Figure 2). The radio-source 28 has been proposed as a $z=2$ radio-galaxy (Maddox et al. 2006), although no radial velocity measurements have been made up to now. We see other two possible scenarios for this radio-source namely it might be (a) a massive binary derived microquasar with superluminal jets; or (b) a kicked-off object (e.g. Gualandris \& Merrit 
2007; Bonning, Shields \& Salviander 2007) associated to a past BHs quasi-merger event at the bulge center of M83.

The 12 minutes co-added image from the observed GMOS-S bands $V$, [OIII], [OIII]C, $\mathrm{H} \alpha, \mathrm{H} \alpha \mathrm{C},[\mathrm{SII}], z, i$, and $\mathrm{CaT}$ is shown in Figure 2. With an average seeing of $0.8^{\prime \prime}$ and considering the background noise from M 83 disk, the approximate detection limit is $\mathrm{m}_{i}=23.5 \pm 0.5$ at $S / N \sim 3$. Although the faintest expected $i$ band apparent magnitude of a radio galaxy at $z=2$ is $\mathrm{m}_{i} \sim 18.5$ nothing is detected as can be seen in Figure 2 . Then, a $z=2$ galaxy have to be absorbed by about 5 magnitudes or more in order to avoid detection in this co-added image. Such level of extinction is not usually expected in the interarm region where the object appears projected.

In the co-added spectra, the detection limit for the continuum of an elliptical galaxy at redshift 2 was $\mathrm{m}_{i}=20.5 \pm 0.8(S / N \sim 3)$, and it was $\mathrm{m}_{i}=22.2 \pm 0.8$ considering the detection of $\mathrm{H}_{\gamma}$ in a QSO at $1<z<2$. Therefore, optical spectroscopy does not confirm too that radio source 28 is a quasar at $z=2$.

\section{Conclusions}

- The luminosity profile of M 83 bulge can be traced by a de Vaucouleurs' law and the inner part can be fitted by a $n=-1 / 2$ Sérsic profile. This characteristic and the IR $(J-K)$ color difference between the periphery and the central part of the bulge, would indicate the presence of a pseudobulge.

- High resolution 3D spectroscopy observations constrain the masses of possible central very dense objects in the $\mathrm{ON}$ and $\mathrm{KC}$, ruling out the presence of a $\mathrm{BH}$ more massive than $10^{6} M_{\odot}$ within these condensations. Our numerical simulations show that ON, KC and $\mathrm{HN}$ will merge forming a single massive core in less than $50 \mathrm{Myr}$.

- Deep optical imaging and spectroscopy on the location of the source Maddox 28 do not confirm the high redshift nature of the source and leave open the question of a possible kick-off remnant pointing to the bulge center.

\section{References}

Adamson, A., Adams, D., \& Warwick, R. 1987, MNRAS 224, 367

Bonning, E., Shields, G., \& Salviander, S. 2007, ApJ 666, L13

Diaz, R., Dottori, H., Mediavilla, E., Aguero, M., \& Mast, D. 2006, New Astron. Rev. 49, 547

Diaz, R., Dottori, H., Aguero, M., Mediavilla, E., Rodrigues, I., \& Mast, D. 2006, ApJ 652, 1122 .

Dottori, H., Diaz, R., Rodrigues,I., Aguero, M., \& Mast, D. 2007, in: V. Karas \& K. Matt (eds.), Black Holes from Stars to Galaxies, Proc. IAU Symp. No. 238 (Cambridge: CUP), p. 277

Elmegreen, D., Chromey, F., \& Warren, A. 1998, AJ 116, 2834

Gualandris, A. \& Merrit, D. 2007, eprint arXiv07080771

Jarret, T., et al. 2003, AJ 125, 525.

Jensen, E., Talbot, R., \& Dufour, R. 1981, ApJ 243, 716

Maddox, L, Cowan, J., Kilgard, R., Lacey, C., Prestwich, A., et al. 2006, AJ 132, 310

Mast, D., Diaz, R., \& Aguero, M. 2006, AJ 131, 1394

Telesco, C. 1988, ARAA 26, 343

Thatte, N., Tecza, M., \& Genzel, R. 2000, A\&SA 364, 47

Parry, I., et al. 2004, SPIE 5492, 1135

Sakamoto, K., Matsushita, S., Peck, A., Wiedner, M., \& Iono, D. 2004, ApJ (Letters) 616, 59

Springel, V. 2005, MNRAS 364, 1105 